\title{
Compensation des risques et Managed Care
}

Ces deux questions n'ont à priori rien à voir l'une avec l'autre. Comme tout le monde le sait la compensation des risques vise primairement à limiter la sélection des risques par les assureurs en se basant sur des critères constitués par l'âge et le sexe. C'est simple et cela fonctionne bien.

Pourquoi dès lors vouloir changer/améliorer le système? De toute évidence les critères actuels ne sont pas suffisants et la sélection des risques continue. C'est même le fonds de commerce de certaines grandes caisses/ groupes pour augmenter leurs parts de marché. Curieusement les bénéficiaires du fonds de compensation des risques sont toujours les mêmes, et curieusement encore ce sont essentiellement les grandes caisses cumulant entre elles 90\% des assurés. Les payeurs sont toujours les mêmes aussi. A entendre santésuisse, ces caisses ont une mauvaise structure d'assurés et il leur appartient de l'améliorer. Seulement voilà, cela prend du temps d'attendre que les assurés vieillissent pour l'équilibrer. Il faut donc continuer cette soi-disant ineptie, qui bien entendu ne serait pas nécessaire s'il n'y avait que les grandes caisses.

La compensation des risques en fonction de l'âge et du sexe est simple et fonctionne bien. Elle permet aussi aux caisses de reporter les déficits de fonctionnement sur les fournisseurs de prestations, car les mêmes critères sommaires de compensation s'appliquent à la clientèle des cabinets.

Les statistiques de santésuisse peuvent alors montrer qu'un médecin sur 8 dévie de manière significative de la moyenne des honoraires par patient de son groupe de spé- cialité indépendamment de la structure de sa patientèle. C'est par conséquent un profiteur. Il doit donc rembourser - quelle manière commode de compenser les risques!

Tout le monde pense, politiques, assureurs et fournisseurs de prestations que le Managed Care est sans doute une bonne chose. Les quelques groupes pilotes en activité en Suisse l'ont bien montré. Cela augmente l'efficience de la prise en charge et permet des économies. Les assureurs s'y intéressent, mais un peu, pas trop. La sélection des risques rapporte davantage. Elle permet au moins d'augmenter les parts de marché.

Sans doute puisqu'il ne faut surtout pas améliorer leur compensation. Le système en fonction de l'âge et du sexe est si simple et fonctionne si bien. Pourquoi vouloir dès lors y introduire encore des critères d'hospitalisation et même de morbidité comme le pensent unanimes tous les économistes de santé de ce pays et quelques parlementaires farfelus? La sélection des risques ne pourrait alors plus rien rapporter et les assureurs seraient obligés de s'intéresser vraiment au Managed Care. Parce que ce serait alors le seul moyen d'être concurrentiel et d'améliorer les parts de marché. Pour autant que les assureurs collaborent avec les médecins et les réseaux de Managed Care - quel changement de paradigme!

Dr Yves Guisan, vice-président de la FMH, Conseiller national 